Published in:

IEEE Robotics and Automation Magazine, vol. 6, no. 4, Dec. 1999, pp. 32-37 (6)

\title{
Research Relevance of Mobile Robot Competitions
}

\author{
Thomas Bräunl \\ Centre of Intelligent Information Processing Systems \\ Dept. of Electrical and Electronic Engineering \\ The University of Western Australia \\ Nedlands, Perth, WA 6907 \\ braunldee. uwa. edu . au \\ www.ee. uwa.edu.au/ braun l
}

\begin{abstract}
A number of international competitions for mobile robots have been created and conducted over the last 20 years. This article discusses three of the arguably most prominent event series. We highlight scope, rules, robot designs and robot evolution over the years for the Micromouse Contest, AAAI Mobile Robot Competition and RoboCup Robot Soccer and discuss their contributions to mobile robot research.
\end{abstract}

\section{Keywords}

mobile robot, competition, micromouse, office navigation, ball collection, robot soccer, RoboCup, robotics research programs.

\section{Introduction}

Mobile robot competitions have been around for over 20 years. They have inspired generations of students, researchers and laypersons alike, while consuming vast amounts of research funding and personal time and effort. Competitions provide a goal together with an objective performance measure, while extensive media coverage allows participants to present their work to a wider forum. So it is time to take a look back and reflect on the research outcome of a number of events.

As the robots in a competition evolved over the years, becoming faster and smarter, so did the competitions themselves. Rule changes and new competitions were proposed, pushing the limits to keep the events challenging for state-of-the-art technology and interesting for research. Robot perception abilities improved tremendously over the years. New and better sensor systems have been added and more sensor data can be evaluated in real time thanks to increased processing speed. In robot competitions this is reflected by postulating very strict environments (e.g. maze) in early competitions, while later competitions take place in more open spaces (e.g. soccer field). Some early robot competitions already played two competing robots against each other, while multiple robots cooperating for a common task have only been introduced a few years ago.

In the next sections, we will take a closer look at three major international mobile robot competitions. In the order of their creation dates these are Micromouse Contest, AAAI Mobile Robot Competition, and RoboCup Robot Soccer. We will highlight 
scope, rules, robot designs and robot evolution over the years. After this, we discuss research contributions of each of the competitions and conclude with an outlook to future events.

\section{Micromouse Contest}

"The stage was set. A crowd of spectators, mainly engineers, were there. So were reporters from the Wall Street Journal, the New York Times, other publications, and television. All waited in expectancy as Spectrum's Mystery Mouse Maze was unveiled. Then the color television cameras of CBS and NBC began to roll; the moment would be recreated that evening for viewers of the Walter Cronkite and John ChancellorDavid Brinkley news shows" [1].

This report from the first "Amazing Micro-Mouse Maze Contest" demonstrates the enormous media interest in the first mobile robot competition in New York in 1977. The academic response was also overwhelming. Over 6000 entries followed the announcement of Don Christiansen [5], who originally suggested the contest.

The task is for a robot mouse to drive from start to goal in the fastest time. Rules changed somewhat over time, in order to allow exploring the whole maze and then compute the shortest path, while also counting exploration time at a reduced factor.

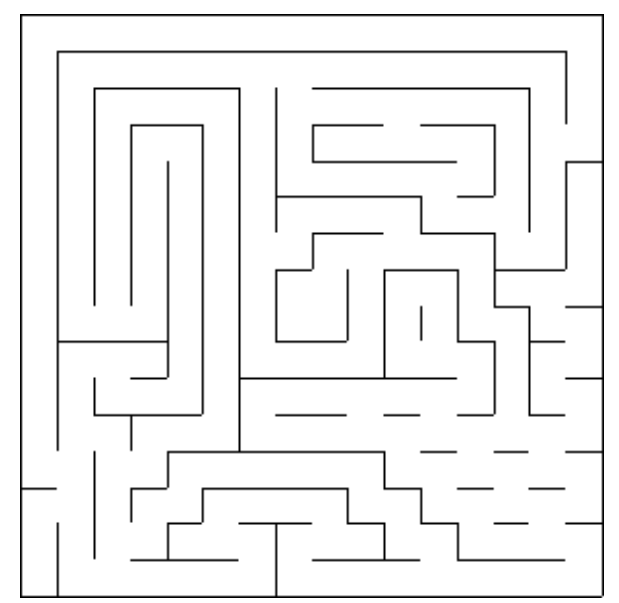

Fig 1. Maze from micromouse contest in London 1986, displayed by EyeSim simulator www.ee. uwa.edu.au/ braunl/eyebot/doc

The first mice constructed were rather simple - some of them contained not even a microprocessor as controller, but were simple "wall huggers" which would find the goal by always following the left (or always the right) wall. A few of these scored even higher than some of the "intelligent" mice, which were mechanically slower.

John Billingsley [2] made the micromouse contest popular in Europe and called for the first rule change: Starting in a corner, the goal should to be in the center and not in another corner, to eliminate the simple electro-mechanical wall huggers. From then on, more intelligent behaviour was required to solve a maze. Virtually all robotics labs 
were building micromice in one form or another at that time - a real micromouse craze was going around the world. All of a sudden, people had a goal and could share ideas with a large number of colleagues who were working on exactly the same problem.
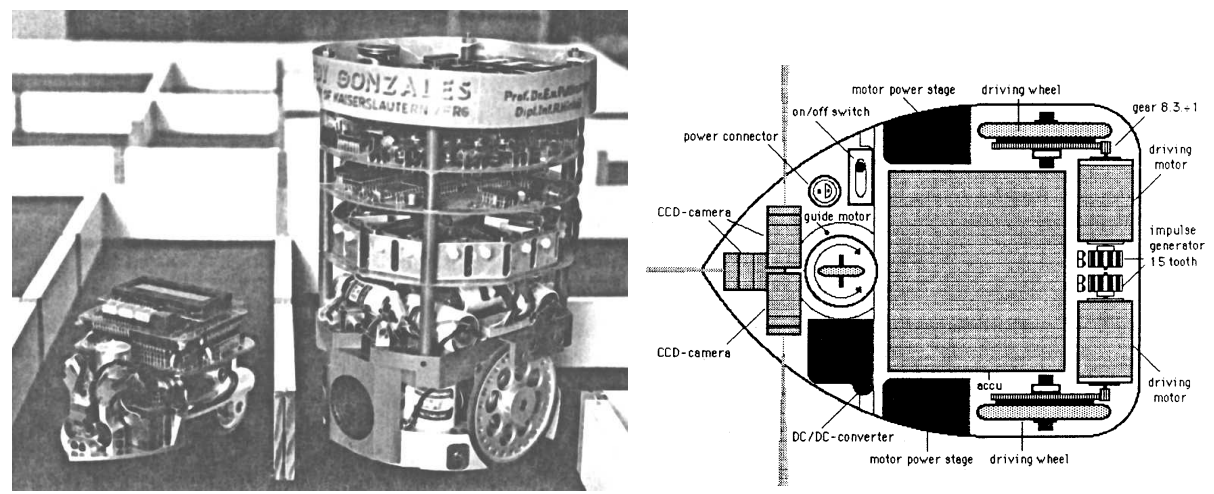

Fig 2. Two generations of micromice from Univ. Kaiserslautern photo/diagram by Ralf Hinkel and Ewald von Puttkamer

Micromouse technology did evolve quite a bit over time, as did the running time. A typical sensor arrangement was using three sensors to detect any walls in front, to the left, and to the right of the mouse. Early mice used simple micro switches as touch sensors, while later on sonar, infrared, or even optical sensors [6] became popular.

While the mouse size is restricted by the maze's wall distance, smaller and especially lighter mice have the advantage of higher acceleration/deceleration and therefore higher speed. Even smaller mice became able to drive in a straight diagonal line instead of going through a sequence of left/right turns, which exist in most mazes.

One of today's fastest mice comes from the Univ. of Queensland (there are still micromouse competitions held in 1999), using three extended arms with several infrared sensors each for reliable wall distance measurement. By and large, it looks as if the micromouse problem has been solved, with the only possible improvement being on the mechanics side, but not in electronics, sensors or software.

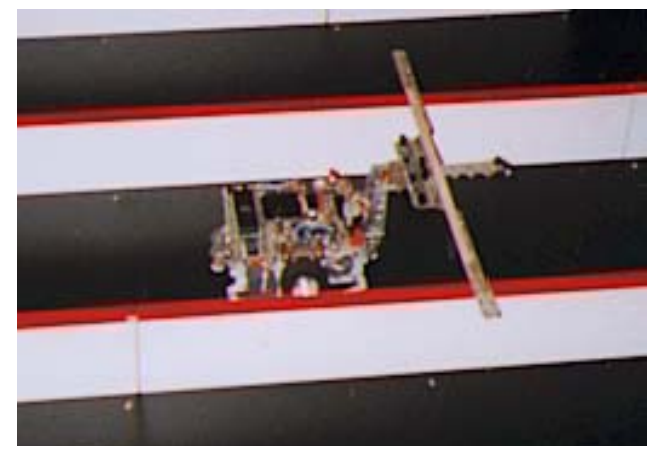

Fig 3. Micromouse from Univ. of Queensland, Gordon Wyeth 


\section{AAAI Mobile Robot Competition}

At the end of the 1980s, the micromouse contest had outlived itself and the robotics research community was losing interest. The problem was solved and did not provide any new challenges. Around that time David Kortenkamp initiated the AAAI Mobile Robot Competition [9]. The first event was held at AAAI 1992 in San Jose, California, where ten robots competed in three events:

- $\quad$ "roaming [a $22 \mathrm{~m} \times 22 \mathrm{~m}$ ] area and avoiding obstacles [cardboard boxes] and wandering judges"

- $\quad$ "searching for 10 distinctive objects [three-inch diameter poles] and then visiting each of the objects"

- $\quad$ "timed race to three of the objects found [in previous task] and then back home"

While the AAAI competitions created new challenges, they were not as media friendly as the micromice - basically because most of these tasks took around $20 \mathrm{~min}$. to complete and the audience could not judge what processes and state changes were going on "inside the robots".

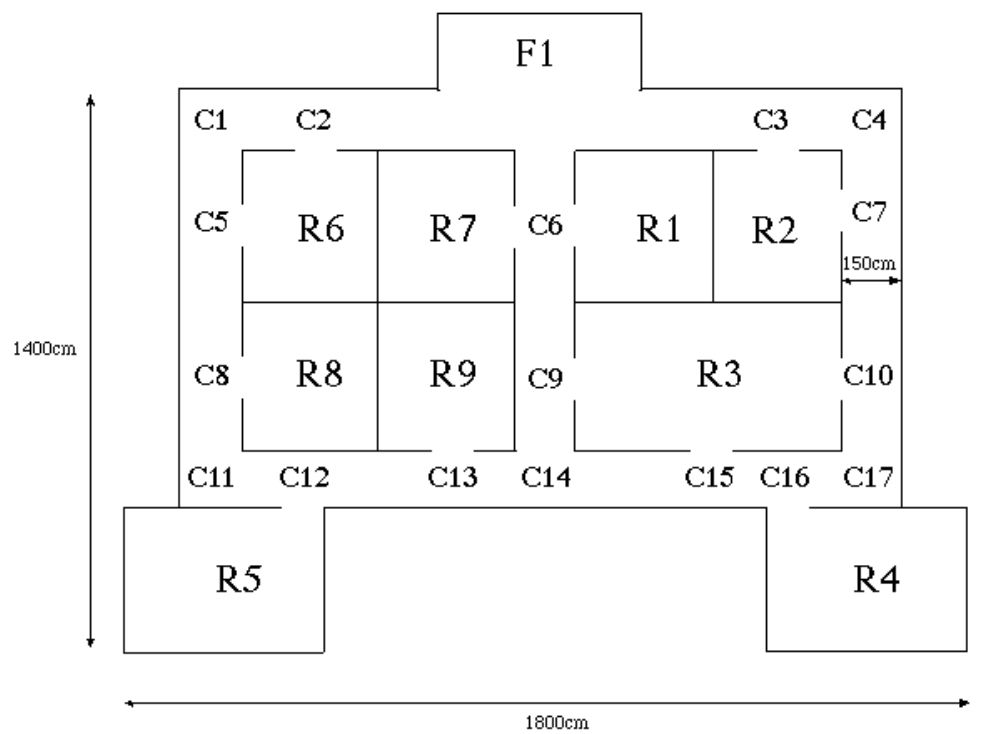

Fig 4. Sample area for "call a meeting" task (from Kortenkamp's web site 1996)

New tasks have been introduced over the years at AAAI. Some competition events were kept over several years and only slightly modified, while other events were modified from year to year. The idea was to always present a new challenge rather than leave one event for too long, so it would not get solved easily. For AAAI 1996 in Portland, Oregon, the tasks were: 
- $\quad$ "call a meeting"

robots have to act as messengers by solving navigation tasks around a (mock)

office environment

- "clean up the tennis court"

robots have to collect tennis balls from a rectangular area and bring them back into a pen

Although the office navigation seems to have a remote resemblance to the micromouse maze, it is quite different. The floor plan is known to the robots before they start, as well as the rooms they have to visit. So it comes down to reliable driving without hitting a wall and correct recognition of (open) doors and hallways. If this sounds almost trivial - it is not, since it takes place in a near-real environment with the typical problems of lighting, (sensor) reflections, etc.

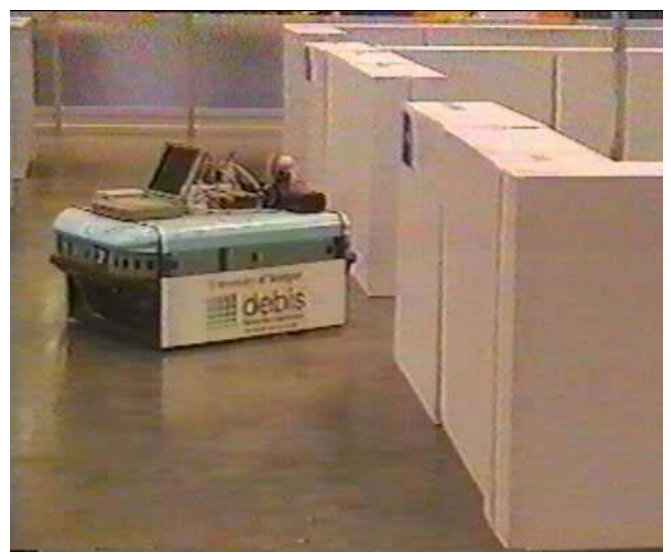

Fig 5. Univ. Stuttgart IPVR "Musketeer" robot in "call a meeting" event, AAAI 96

While "call a meeting" could be solved by using sonar or laser range sensors, "clean up the tennis court" required the use of image processing in order to detect and track the tennis balls. "Squiggle balls" (balls with a motor inside to make them follow unpredictable paths) were added as an extra difficulty. Traditional planner-based programs can be used to solve the office navigation task. The ball collection task on the other hand allows a choice of either planning or behaviour-based approaches. Newton Labs won this event in 1996 with a behaviour-based approach. The robot would just search for a yellow tennis ball with its color camera, when detected rush towards it, grab it, and then search for the pen area (marked by a blue bar) to return the ball. It did not make use of cartesian coordinates and had no internal model of its position or orientation, although it would have been advantageous to do so. Other approaches, like Univ. Stuttgart [4], used a sweeping pattern or area covering approach, employing vision to catch individual stray balls. 

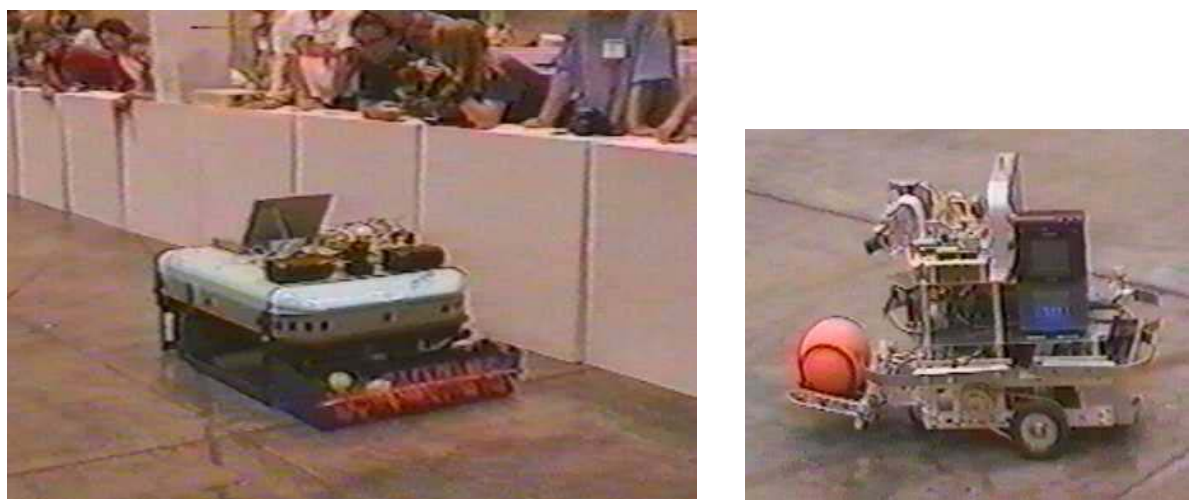

Fig 6. Ball collecting robots from Univ. Stuttgart IPVR and Newton Labs

\section{RoboCup}

"The Robot World Cup Initiative (RoboCup) is an attempt to foster AI and intelligent robotics research by providing a standard problem where wide range of technologies can be integrated and examined. For this purpose, RoboCup chose to use soccer game, and organize RoboCup: The Robot World Cup Soccer Games and Conferences. In order for a robot team to actually perform a soccer game, various technologies must be incorporated including: design principles of autonomous agents, multi-agent collaboration, strategy acquisition, real-time reasoning, robotics, and sensor-fusion. RoboCup is a task for a team of multiple fast-moving robots under a dynamic environment. RoboCup also offers a software platform for research on the software aspects of RoboCup." (from the RoboCup web site www. robocup. org)

So robot soccer brings mobile robots to spectator sport. Huge media interest was the result after Hiroaki Kitano created this competition in 1997 [7],[8]. The competition actually comprises three leagues at the moment:

- Small size league (robots of up to $180 \mathrm{~cm}^{2}$ floor area)

- Medium size league (robots of up to $2000 \mathrm{~cm}^{2}$ floor area)

- $\quad$ Simulator league

A planned large size league has been dropped, while legged robot soccer leagues and commentator leagues are currently being discussed.

Robot soccer teams play five-a-side soccer with rules that are freely adapted from FIFA soccer. Since there is a boundary around the playing field in both "real" robot events, the game is actually closer to ice hockey. The big challenge is that not only reliable image processing has to be performed in real time, but also that a team of five robots/actors has to be organised. In addition, there is an opponent team which will change the environment (e.g. kick the ball) and thereby render one's own action plans useless if one is too slow.

The small size league plays on a field the size of a ping-pong table. The use of external sensors is allowed, especially an overhead camera, which facilitates absolute 

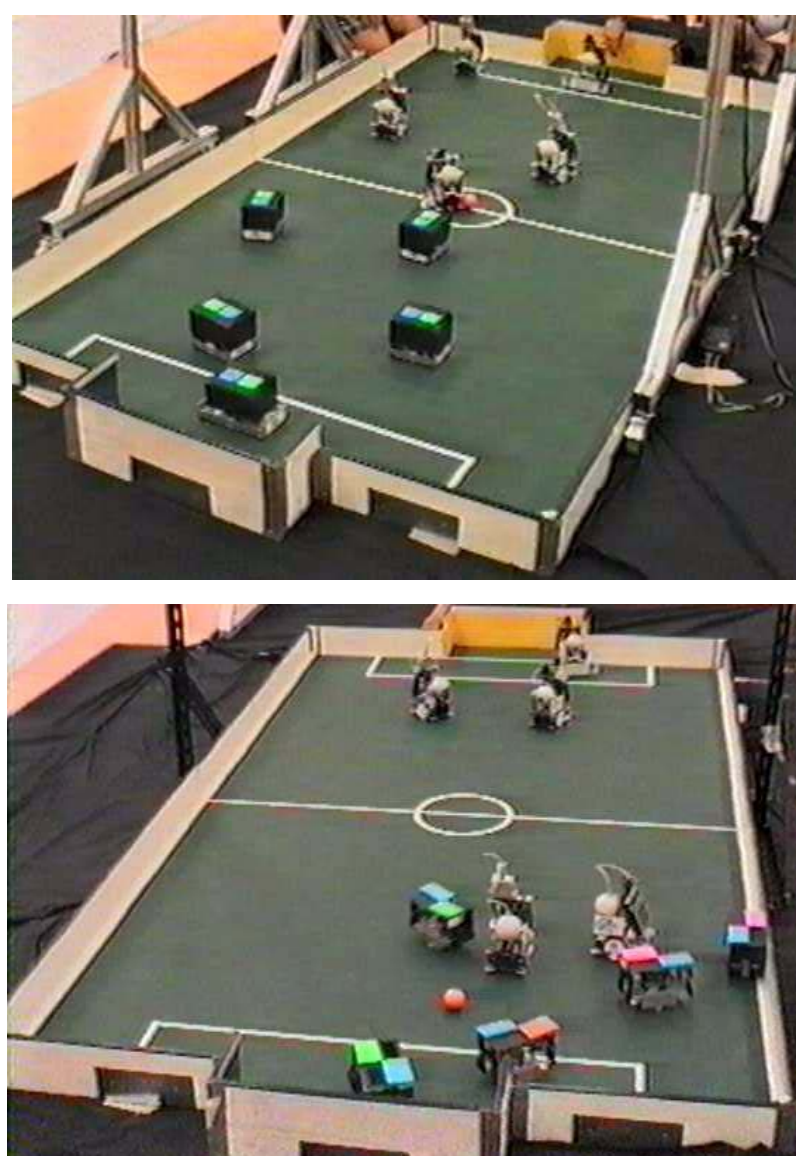

Fig 7. RoboCup match setup and during play,

CIIPS Glory (Australia) vs. Lucky Star and Lilliputs (both Singapore)

positioning of robots (own and opponent), ball and goals. Most of the teams so far follow this approach, which requires a wireless transmission from the image processing workstation to the robots. This led to the development of remote controlled units (one team actually used remote controlled toy cars) rather than mobile robots with local sensors. Most teams have no sensors at all on the robots, and communication is a oneway broadcast from the workstation to the robots. An exception is the CIIPS Glory robot soccer team from UWA [3], which uses local cameras and on-board computation without a host workstation.

The medium size league does not allow global vision, so the participating robots are required to have local sensors and local intelligence, however, remote control from powerful workstations is still allowed. Many teams use off-the-shelf mobile robots driven by laptop computers. This makes it a costly exercise, which not all interested institutes are capable or willing to fund. In addition, the large field size of up to $10 \mathrm{~m} \times 7 \mathrm{~m}$ makes it hard for many teams to find appropriate practice/lab space. 


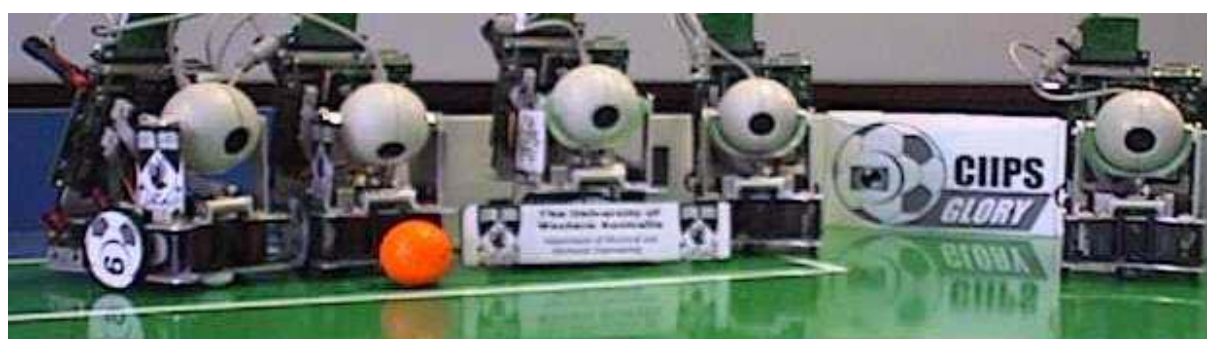

Fig 8. Robot Soccer Team CIIPS Glory from UWA

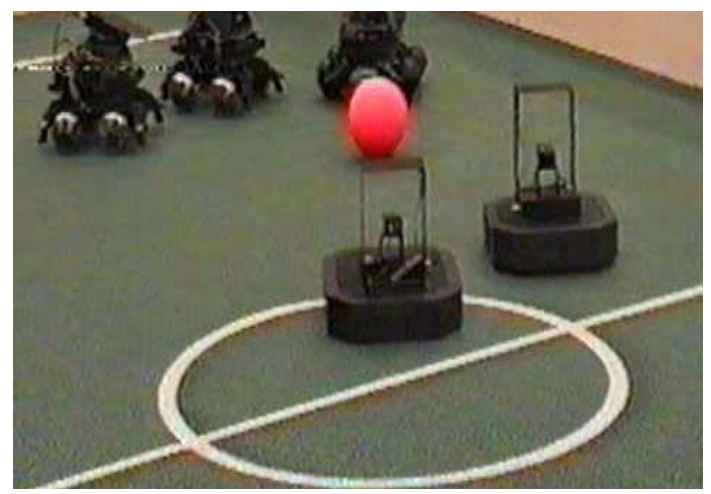

Fig 9. Robcup medium size league, Singapore 1998

\section{Research Contributions}

One of the frequent disappointments of robot competitions is that enormous research efforts are being reduced to performance in a particular event and cannot be appreciated adequately. Adapting from the home lab environment to the competition environment turns out to be quite tricky, and many programs are not as robust as their authors had hoped. On the other hand, the actual competitions are only one part of the event. Most competitions are embedded in conferences and encourage participants to present the research behind their competition entry, giving them the right forum to discuss related ideas.

At first glance competitions seem to be an objective measure for good research, but this is certainly not true. Several aspects have to be taken into account, especially how much of a system is the team's own research effort and how much is bought-in technology. Several mobile robot companies already offer specialized products for RoboCup or AAAI competitions, including both hardware and software. So money can make quite a difference here, which makes it almost impossible to evaluate and honor the actual research contribution.

Most mobile robot competitions involve interesting research problems which could be studied in their own right. The following (non-exhaustive) list mentions just a few of them: 
- $\quad$ robot soccer

- $\quad$ multi-robot / multi-agent scenarios

- cooperative work

- communication

- $\quad$ real time image tracking

- object identification

- $\quad$ sensor fusion

- planning

- office navigation

- planning

- $\quad$ robust (sonar) sensor processing

- obstacle avoidance

- human-robot interaction

- ball collection

- mechanics for ball collection

- $\quad$ path planning

- $\quad$ robust sensor processing

- $\quad$ real time image tracking

Mobile robot competitions brought progress to the field by inspiring the people who worked on them and by continuously pushing the limits of what is possible. Through robot competitions, progress has been achieved in mechanics, electronics, and algorithms. We have seen technology changes in sensor development from tactile sensors over sonar and infrared sensors to radar and optical sensors.

Simple vision systems used for RoboCup are capable of performing image processing at a rate of a few frames per second. These teams cannot compete against real-time vision systems with PAL video frequency of $25 \mathrm{fps}(50 / 2)$, while some teams reach image processing and command update frequencies of up to $60 \mathrm{fps}$.

It is interesting to see that smaller robots tend to perform better - except in robot soccer, where blocking the way is part of the game. This holds not only for the Micromouse Contest where a smaller mouse has an obvious path advantages for being able to drive diagonally, but also for both AAAI'96 competition tasks. Smaller robots have less mass and can therefore react faster. However, they have the disadvantage of much tighter payload and battery restrictions, which severely limit the sensor and computing equipment that can be carried (unless wireless transmissions are allowed).

Micromouse and AAAI (so far) have been rather static problems, which were basically "solved" at some stage. RoboCup on the other hand means playing an opponent, which will evolve as well over the years, so it will hopefully remain interesting for quite a while. The three mobile robot competitions presented here are not the only ones. There are a number of other active competitions and new ideas are always being discussed.

It is hard to say what changes future mobile robot competitions will bring. But interpolating from the past, competitions will probably have groups of robots cooperate in less specialized environments, so they will eventually perform without restrictions in 
unchanged office or home environments. More challenging tasks will require more sophisticated image processing than the present color identification. New robot control models may be developed and implemented, but cannot and should not be forced by a competition. After all, it is an almost impossible task to design the "optimal" mobile robot competition, which covers all currently valid research areas, discourages industrial solutions and is still fun to students.

\section{Conclusion}

Mobile robot competitions are a big opportunity for robotics research. They create student interest and inspire them to work dedicated on their projects. Competitions create media interest and may even generate additional funds from external sources. The practical solutions students find for all the small problems on their way to a competition will teach them more skills than a lecture ever can.

So it may actually be a good idea to participate in a robot competition, provided that the individual research goals are covered by the particular event. It can be seen as a useful "umbrella", which provides a meaningful application to a number of more or less related robotics research topics.

However, we must be careful not to confuse a competition with research. The objective for competitions must remain to interest students in a particular research area, to foster or initiate research in particular topics in mechanics, electronics or algorithm design, and to build a reliably working (fault-tolerant) solution to a given problem. A competition must not be "l'art pour l'art", it can only be part of a larger, well organised research program.

\section{References}

1. Roger Allan, The amazing micromice: see how they won, IEEE Spectrum, Sep. 1979, vol. 16, no. 9, pp. 62-65

2. John Billingsley, Micromouse - Mighty mice battle in Europe, Practical Computing, Dec. 1982, pp. 130-131

3. T. Bräunl, B. Graf, Robot Soccer with Local Vision, Pacific Rim International Conference on Artificial Intelligence, Singapore, Nov. 1998, pp. 14-23

4. T. Bräunl, M. Kalbacher, P. Levi, G. Mamier, CoMRoS: Cooperative Mobile Robots Stuttgart, Proceedings of the Thirteenth National Conference on Artificial Intelligence, AAAI Press, Portland Oregon, Aug. 1996, pp. 1351

5. C. Christiansen, Announcing the amazing micromouse maze contest, IEEE Spectrum, May. 1977, vol. 14, no. 5, pp. 27

6. Ralf Hinkel, Low-Level Vision in an Autonomous Mobile Robot, EUROMICRO 1987; 13th Symposium on Microprocessing and Microprogramming, Portsmouth, England; Sep. 1987; pp. 135-139

7. H. Kitano, M. Tambe, P. Stone, M. Veloso, S. Coradeschi, E. Osawa, H. Matsubara, I. Noda, M. Asada, The RoboCup synthetic agent challenge 97, Proc. of the Fifteenth Intl. Joint Conf. on Artificial Intelligence, San Francisco CA, 1997, pp. 24-29 
8. H. Kitano, M. Asada, I. Noda, H. Matsubara, RoboCup: Robot World Cup, IEEE Robotics and Automation Magazine, vol. 5, no. 3, Sep. 1998, pp. 30-36

9. David Kortenkamp, M. Huber, C. Cohen, U. Raschke, C. Bidlack, C. Congdon, F. Koss, T. Weymouth, Winning the AAAl Robot Competition, Proceedings AAAI '93, National Conference on Artificial Intelligence, San Jose, California, pp. 858-859 (2)

\section{Short Author Biography}

Thomas Bräunl is associate professor at The University of Western Australia, Perth, Department of Electrical and Electronic Engineering, where he directs the mobile robot lab. He received M.S. degrees from Kaiserslautern University in 1986 and the University of Southern California, Los Angeles, in 1987, and a Ph.D. and Habilitation in Computer Science from Stuttgart University in 1989 and 1994, respectively.

Prof. Bräunl works in the areas of mobile robots, vision, graphics, and concurrency. He has published several textbooks and created the EyeBot mobile robot family.

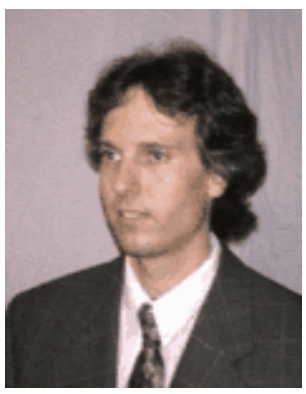

